\title{
Acesso e uso de serviços de Fonoaudiologia em Porto Alegre, Brasil: estudo populacional
}

\author{
Access and use of speech-language therapy services in Porto Alegre, \\ Brazil: a population-based study
}

Rafaela Soares Rech (https://orcid.org/0000-0002-3207-0180) ${ }^{1}$

Patrícia Távora Bulgarelli (https://orcid.org/0000-0001-9902-8019) ${ }^{2}$

Aline Macarevich Condessa (https://orcid.org/0000-0002-8129-4324) ${ }^{3}$

Camila Mello dos Santos (https://orcid.org/0000-0001-5354-3699) ${ }^{2}$

Juliana Balbinot Hilgert (https://orcid.org/0000-0002-2204-1634) ${ }^{1}$

Bárbara Niegia Garcia de Goulart (https://orcid.org/0000-0002-2502-5883) ${ }^{1}$

${ }^{1}$ Programa de PósGraduação em

Epidemiologia, Faculdade de Medicina, Universidade Federal do Rio Grande do Sul (UFRGS). R. Ramiro Barcelos 2492, Santa Cecília. 90035-004 Porto Alegre RS Brasil.

bngoulart@gmail.com ${ }^{2}$ Programa de Pós-

Graduação em Saúde Coletiva, Faculdade de Enfermagem, UFRGS. Porto Alegre RS Brasil.

${ }^{3}$ Programa de Pós-

Graduação em Odontologia, Faculdade de Odontologia, UFRGS. Porto Alegre RS Brasil.

\begin{abstract}
To estimate the prevalence of access and use of speech-language therapy services and identify the variables associated with access. Cross-sectional population-based study. The sample consisted of adult individuals living in Porto Alegre, southern Brazil. The data were collected using an instrument constructed with domains of national research questionnaires, with a module on speech-language therapy. The outcome was the access to a speech-language therapist. Poisson regression with robust variance was used to calculate Prevalence Ratios with 95\% confidence intervals. A total of 214 people participated in the study, of which $67.3 \%(n=144)$ were female. The mean age was 54.28 ( $S D \pm 18.83)$ years. Fifty-six (26.2\%) people mentioned the need for speech -language therapy consultation. All 56 subjects were able to perform speech-language therapy, of which $69.4 \%(n=39)$ in private practice and $19.6 \%$ ( $n=11)$ used healthcare insurance plans at partnering providers. In the final model, the highest prevalence of access was associated with $\mathrm{fe}$ male $(P R=1.09,95 \% C I 1.01-1.18)$ and had some deficiency $(P R=1.09,95 \%$ CI1.03-1.17). Access to a speech-language therapist is more frequent in private services. It is observed that women and the disabled individuals have a higher prevalence of access to speech-language therapist.
\end{abstract}

Key words Access to Health Services, Speech-language therapy, Prevalence
Resumo O objetivo deste artigo é estimar a prevalência de acesso e uso dos serviços de Fonoaudiologia e identificar as variáveis associadas ao acesso. Estudo transversal de base populacional. A amostra foi composta de indivíduos adultos residentes em Porto Alegre/RS. Os dados foram coletados a partir de um instrumento construído com dominios de questionários de pesquisas nacionais, com um módulo sobre Fonoaudiologia. O desfecho estudado foi o acesso ao fonoaudiólogo. Regressão de Poisson com variância robusta foi utilizada para cálculo de Razões de Prevalência com intervalos de confiança de 95\%. Aceitaram participar deste estudo 214 pessoas, das quais 67,3\% ( $n$ =144) eram do sexo feminino. A média de idade foi de 54,28 $( \pm 18,83)$ anos. Referiram necessidade de consulta fonoaudiológica 56 (26,2\%) pessoas. Todos os 56 indivíduos conseguiram realizar atendimento fonoaudiologico, dos quais $69,4 \%(n=$ 39) em consultório particular e 19,6\% $(n=11)$ em consultório conveniado ao plano de saúde. No modelo final, maior prevalência de acesso foi associada ao sexo feminino ( $R P=1,09$; IC95\% 1,01$1,18)$ e possuir alguma deficiência $(R P=1,09$; IC95\% 1,03-1,17). O acesso ao fonoaudiólogo é mais frequente de forma privada. Observa-se que as mulheres e deficientes possuem maior prevalência de acesso ao fonoaudiólogo.

Palavras-chave Acesso aos Serviços de Saúde, Fonoaudiologia, Prevalência 


\section{Introdução}

O acesso à saúde refere-se à utilização do serviço atendendo a necessidade individual ${ }^{1}$. Acesso é o uso real de serviços de saúde e tudo o que facilita ou impede sua utilização, obtendo o serviço adequado no momento certo para promover melhores resultados de saúde ${ }^{2}$. A utilização dos serviços de saúde é consequência de uma interação de fatores, entre eles a percepção desta sob a ótica do usuário e a oferta disponível do serviço ${ }^{3}$.

Estudos que analisaram o acesso e o uso de serviços de saúde retratam vulnerabilidades e desigualdades sociais, resultantes das condições sociais dos indivíduos e dos locais onde residem $^{4-6}$. Sabe-se que o acesso está diretamente relacionado à oferta, e dificuldades nesse acesso relacionam-se às particularidades dos sistemas $\mathrm{e}$ serviços de saúde, envolvendo aspectos econômicos, políticos, técnicos e organizativos ${ }^{7,8}$. Investigações de base populacional que abordam o uso de serviços de saúde são essenciais para descrever tendências e frequências. Os resultados obtidos tornam possível o conhecimento do real acesso e uso de serviços de determinada população, viabilizando a produção de bases para adequado planejamento, (re)formulação e gerenciamento de políticas de saúde ${ }^{9,10}$.

$\mathrm{O}$ acesso limitado aos cuidados especializados é um importante obstáculo para os cuidados em saúde. No Canadá, descobriu-se que $41 \%$ dos usuários dos serviços de saúde esperavam mais de 2 meses para uma consulta com um profissional específico. Além disso, nos Estados Unidos, $39 \%$ dos indivíduos que possuíam rendimentos abaixo da média referiram dificuldades no acesso aos profissionais especializados, sendo a maior queixa relacionada ao custo ${ }^{11,12}$. No Brasil, o acesso aos serviços de saúde vem aumentando, tanto para os usuários exclusivos do Sistema Único de Saúde (SUS) quanto para os usuários de plano de saúde ou serviço particular, mas as desigualdades ainda persistem ${ }^{13,14}$.

Levantamentos epidemiológicos de base populacional sobre distúrbios da comunicação humana e acesso aos serviços de Fonoaudiologia são escassos. O único levantamento brasileiro encontrado na literatura indexada estimou prevalência de 30,8\% de alterações fonoaudiológicas na população adulta ${ }^{15,16}$. A Fonoaudiologia se propõe a desenvolver uma ampla gama de ações, abrangendo a proteção específica, o diagnóstico, o tratamento precoce e a reabilitação dos distúrbios da linguagem oral e escrita, audição, voz, motricidade orofacial e disfagia orofaríngea; logo, existem diversas demandas da população para o atendimento com um fonoaudiólogo.

A escassez de fonoaudiólogos acessíveis a qualquer população é um desafio a ser superado mundialmente ${ }^{17}$. Estratégias têm sido desenvolvidas para favorecer os cuidados em Fonoaudiologia de forma universal e integral para a população ${ }^{18}$. Assim, é importante que se direcionem esforços para a investigação das variáveis associadas ao acesso e a necessidade dos cuidados em Fonoaudiologia, visando identificar os indivíduos mais suscetíveis ao não acesso ao fonoaudiólogo.

Este estudo, de base populacional, é inédito em sua proposição. O seu objetivo é estimar a prevalência de acesso e uso dos serviços de Fonoaudiologia e identificar as variáveis associadas ao acesso.

\section{Metodologia}

Trata-se de um estudo transversal de base populacional, aninhado a um projeto maior realizado em parceria com a prefeitura de Porto Alegre, que objetiva analisar o acesso aos serviços de atenção primária em saúde ${ }^{19}$. A amostra foi composta de indivíduos adultos, com idade mínima de 18 anos residentes há pelo menos 12 meses nas áreas cobertas pela rede pública de atenção primária em saúde no município de Porto Alegre/RS entre os anos de 2016 e 2017.

A amostra do presente estudo foi estimada considerando a prevalência de $42,4 \%$ do atributo acesso, conforme aferido entre usuários de serviços de atenção primária à saúde e moradores da área coberta pela Gerência Distrital/GD Partenon-Lomba do Pinheiro no ano de 2012, medida por meio do Primary Care Assessment Tool (PCATool $)^{20}$. Foi utilizado um erro de $9 \%$ e, portanto, estimado um intervalo de 0,33-0,51. Das oito gerências distritais do município, três gerências foram selecionadas para o estudo. A seleção das gerências distritais se deu de forma aleatória simples (sorteio). As gerências distritais sorteadas foram a Gerência Centro com população de 277.321 habitantes, a Gerência Partenon-Lomba do Pinheiro com população de 172.928 habitantes e a Gerência Restinga-Extremo Sul com população de 93.509 habitantes. Em cada gerência, adicionou-se 20\% para eventuais recusas e um deff de 1,5 de modo a resguardar precisão, considerando a estrutura do plano amostral. Seguindo a proporcionalidade na distribuição por gerência distrital, o tamanho final da amostra das três gerências distritais escolhidas foi de 214 respondentes (Figura 1). 


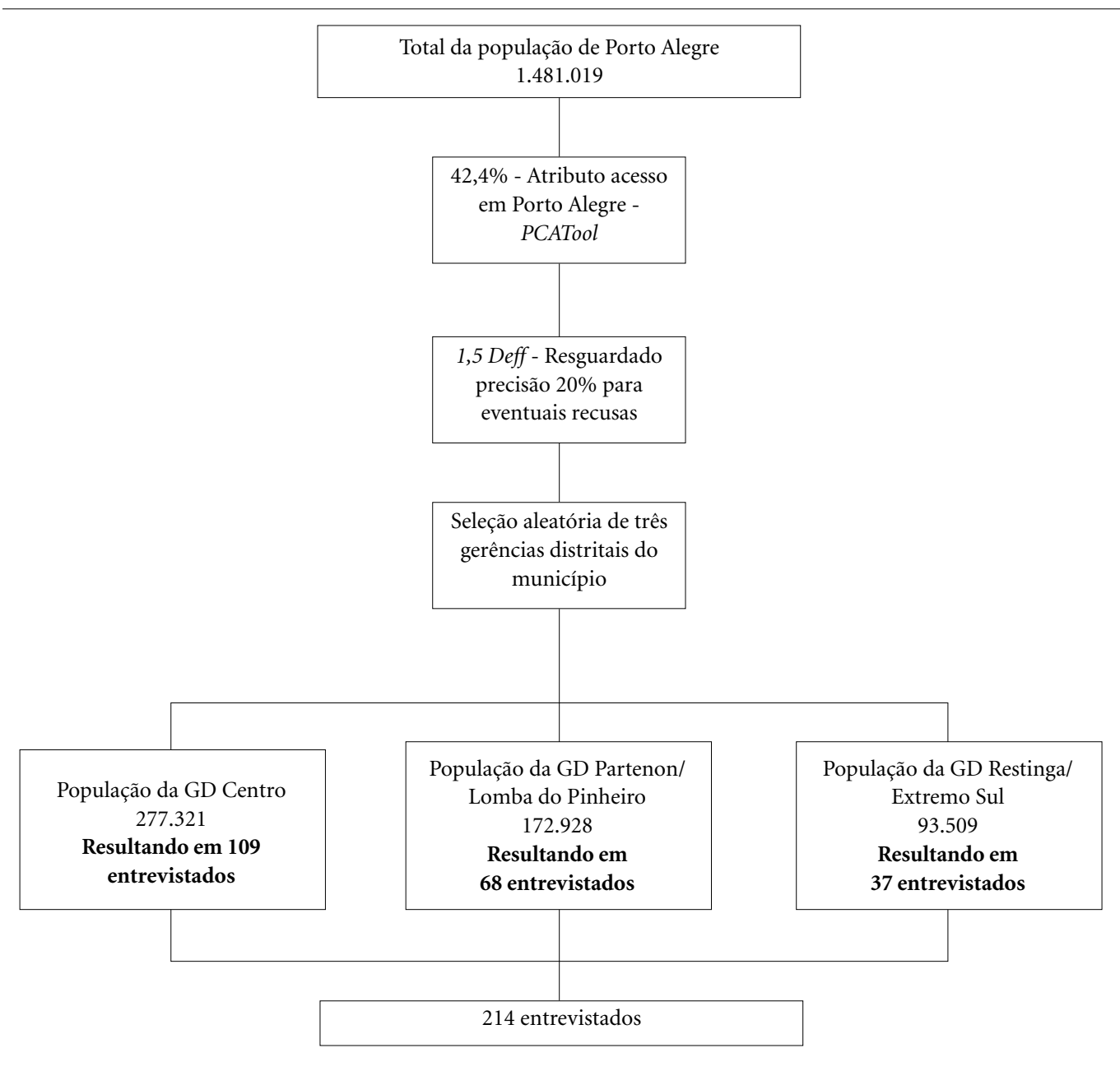

Figura 1. Fluxograma de composição da amostra estudada.

Durante a coleta, dentro de cada gerência, foram selecionadas com início aleatório as casas das famílias e, de cada casa, foi entrevistado um usuário. Nos casos de mais de um adulto na residência, realizou-se um sorteio. Incluiu-se na amostra indivíduos que residissem no território sorteado há pelo menos 12 meses. A amostragem de domicílios se deu de forma sistemática, de modo a garantir que todos os domicílios cobertos pelos serviços de saúde tivessem a mesma chance de entrar para o estudo. A seleção dos domicílios se deu da seguinte forma: $1^{\circ}$ ) O coordenador de campo possuía um mapa do setor censitário a ser visitado e a ordem das quadras numeradas; $\left.2^{\circ}\right)$ Em cada quadra no mapa, uma das esquinas estava destacada, de acordo com sorteio prévio; $\left.3^{\circ}\right)$ Após situar-se na esquina delimitada, de frente às casas, o coordenador, caminhando pela rua da esquerda, localizou a primeira casa cuja porta de entrada estivesse orientada para a rua; $4^{\circ}$ ) A partir da identificação desta casa, sorteou-se qual seria a primeira casa a ser visitada com uma moeda, sendo cara equivalente ao domicílio número 1 e coroa ao domicílio número 2; 5) Após abordagem na primeira casa sorteada, seguiu-se adiante pulando o próximo domicílio existente, até completar a meta de amostragem ou chegar à esquina inicial.

Os dados foram coletados em tablet a partir de um questionário construído com blocos específicos dos questionários da Pesquisa Nacional por Amostra de Domicílios/PNAD (Dados de Identificação, Características de Educação e Rendimentos Domiciliares), Pesquisa Nacional de Saúde/PNS (Informações do Domicílio, Cobertura de Plano de Saúde, Visitas Domiciliares 
de Equipes de Saúde, Estilo de Vida, Utilização de Serviços de Saúde e Saúde Bucal) e do PCATool (Grau de Afiliação, Acesso de Primeiro Contato - Utilização e Acesso de Primeiro Contato - Acessibilidade). Foi incluído um módulo sobre Fonoaudiologia com questões sobre avaliação audiológica, necessidade de atendimento fonoaudiológico, motivo do atendimento, acesso à consulta, local de atendimento, necessidade de tratamento e acesso ao tratamento. Este questionário foi previamente testado em um estudo piloto, realizado entre agosto e setembro de 2016 em 11 indivíduos. Posteriormente ao estudo piloto houve ajustes no questionário com vistas a otimização do tempo de duração e o entendimento dos entrevistados.

Todos os entrevistadores foram devidamente treinados. O desfecho foi criado a partir da variável que identificou o acesso ao atendimento fonoaudiológico, categorizado em sim e não, originada a partir dos seguintes questionamentos aos entrevistados: "Você já precisou se consultar com um fonoaudiólogo (breve explicação da profissão pelo entrevistador)?” (sim; não) e "Você conseguiu ter acesso a essa consulta?" (sim; não). Foi considerado como acesso positivo ao fonoaudiólogo quando o entrevistado respondeu sim para as duas perguntas. As variáveis exploratórias utilizadas foram: sexo (masculino; feminino), idade (em anos: 18-39; 40-59; 60 ou mais), cor/raça (branca; outra), situação conjugal (em relacionamento; solteiro), escolaridade (até ensino fundamental; até ensino médio; ensino superior ou mais), auto relato do diagnóstico de doença crônica, mental ou física (não; sim), possuir algum tipo de deficiência (física, sensorial ou mental: não; sim), utilizar a unidade de saúde do município (sim; não) e cobertura por plano de saúde (não; sim).

Os dados foram analisados no software SPSS v.21 (Chicago: SPSS Inc). O teste do Qui-quadrado foi utilizado para avaliar diferenças nas variáveis estudadas, e quando este violou seus pressupostos utilizou-se o Teste Exato de Fisher, ambos com nível de 5\% de significância. Regressão de Poisson com variância robusta foi utilizada para cálculo de Razões de Prevalência (RP) brutas e ajustadas e seus respectivos intervalos de confiança de $95 \%$. Para a análise multivariável, considerou-se a categoria ter acesso positivo ao fonoaudiólogo como referência para ajuste e interpretação dos dados. Para o modelo ajustado, foram incluídas apenas as variáveis teoricamente relevantes, com valor de $\mathrm{p}<0,3$. O ajuste do modelo foi avaliado com o teste de Deviance.
O presente estudo foi aprovado pelo Comitê de Ética em Pesquisa da Secretaria Municipal de Saúde de Porto Alegre e, também, pelo Comitê de Ética em Pesquisa da Universidade Federal do Rio Grande do Sul.

\section{Resultados}

Foram abordadas 655 pessoas e 214 aceitaram participar da pesquisa. As recusas foram relacionadas à insegurança (receber os entrevistadores em casa), e falta de tempo para participar da pesquisa. Dos respondentes, $67,3 \%(n=144)$ eram do sexo feminino. A média de idade foi de 54,28 $( \pm 18,83)$ anos e a maioria preferiu não relatar a renda mensal. Referiram já ter realizado avaliação audiológica $54,2 \%(n=116)$ das pessoas e $26,2 \%$ $(\mathrm{n}=56)$ referiram necessidade de consulta fonoaudiológica em algum momento.

Os motivos para a necessidade de consulta fonoaudiológica foram audição $(66,1 \% ; \mathrm{n}=37)$, fala $(12,5 \% ; n=7)$, voz $(8,9 \% ; n=5)$, deglutição $(8,9 \% ; n=5)$, fala-leitura/escrita-audição $(1,8 \%$; $\mathrm{n}=1)$ e fala-audição $(1,8 \% ; \mathrm{n}=1)$. Todos os 56 indivíduos que necessitavam conseguiram realizar atendimento fonoaudiológico, dos quais $69,4 \%$ ( $\mathrm{n}=39$ ) foram em consultório particular com financiamento particular, 19,6\% $(\mathrm{n}=11)$ foram em consultório conveniado ao plano de saúde, $3,6 \%(\mathrm{n}=2)$ foram em unidade de saúde, 3,6\% $(\mathrm{n}=2)$ foram em hospital público, $1,8 \%(\mathrm{n}=1)$ foi em clínica-escola de universidade e 1,8\% (n =1) foi no serviço social do comércio. Dentre os que realizaram consulta fonoaudiológica, $57,1 \%$ $(\mathrm{n}=32)$ realizaram tratamento fonoaudiológico, $39,3 \%(\mathrm{n}=22)$ referiram não haver necessidade de tratamento, $3,1 \%(\mathrm{n}=1)$ desistiu e $3,1 \%(\mathrm{n}=$ 1) referiu ser caro.

O acesso ao fonoaudiólogo foi predominantemente por mulheres $(57,1 \% ; \mathrm{n}=32)$, indivíduos com 60 anos ou mais $(51,8 \% ; n=29)$, cor branca $(78,8 \% ; n=44)$, com diagnóstico de doença crônica, mental ou física $(58,9 \% ; n=33)$ e com posse de plano de saúde $(67,9 \% ; n=38)$. Não foram observadas diferenças significativas para o acesso ao atendimento fonoaudiológico em função do sexo $(p=0,069)$, entre as diferentes faixas etárias ( $p=$ $0,529)$, cor/raça $(\mathrm{p}=0,305)$, situação conjugal ( $\mathrm{p}$ $=0,788)$, escolaridade $(p=0,962)$, diagnóstico de alguma doença crônica, física ou mental $(\mathrm{p}=$ $0,457)$, utilização da unidade de saúde do município $(\mathrm{p}=154)$ e adesão a plano de saúde $(\mathrm{p}=$ $0,153)$, enquanto que possuir deficiência ( $\mathrm{p}=$ $0,026)$ foi significativamente associado (Tabela 1 ). 
Tabela 1. Descrição da amostra estudada através de inquérito domiciliar em uma população de adultos na cidade de Porto Alegre, 2017.

\begin{tabular}{|c|c|c|c|}
\hline \multirow{2}{*}{ Variáveis } & \multicolumn{2}{|c|}{ Acesso ao fonoaudiólogo } & \multirow[t]{2}{*}{ p-valor } \\
\hline & Sim & Não & \\
\hline Sexo & & & 0,069 \\
\hline Masculino & $24(42,9 \%)$ & $46(29,1 \%)$ & \\
\hline Feminino & $32(57,1 \%)$ & $112(70,9 \%)$ & \\
\hline Idade & & & 0,529 \\
\hline 18-39 anos & $12(21,4 \%)$ & $45(28,5 \%)$ & \\
\hline 40-59 anos & $15(26,8 \%)$ & $43(27,2 \%)$ & \\
\hline 60 anos ou mais & $29(51,8 \%)$ & $70(44,3 \%)$ & \\
\hline Cor/Raça & & & 0,305 \\
\hline Branca & $44(78,8 \%)$ & $113(71,5 \%)$ & \\
\hline Outra & $12(21,2 \%)$ & $45(28,5 \%)$ & \\
\hline Situação conjugal & & & 0,788 \\
\hline Em relacionamento & $24(42,9 \%)$ & $71(44,9 \%)$ & \\
\hline Solteiro & $32(57,1 \%)$ & $87(55,1 \%)$ & \\
\hline Escolaridade & & & 0,962 \\
\hline Até Ensino Fundamental & $11(19,6 \%)$ & $33(20,9 \%)$ & \\
\hline Até Ensino Médio & $23(41,1 \%)$ & $66(41,8 \%)$ & \\
\hline Ensino Superior ou mais & $22(39,3 \%)$ & $46(37,3 \%)$ & \\
\hline Diagnóstico de doença crônica, mental ou física & & & 0,457 \\
\hline Não & $23(41,1 \%)$ & $74(46,8 \%)$ & \\
\hline Sim & $33(58,9 \%)$ & $84(53,2 \%)$ & \\
\hline Possuir deficiência & & & 0,026 \\
\hline Não & $40(71,4 \%)$ & $86(54,4 \%)$ & \\
\hline Sim & $16(28,6 \%)$ & $72(45,6 \%)$ & \\
\hline Utilizar a unidade de saúde do município & & & 0,154 \\
\hline Não & $24(42,9 \%)$ & $51(32,3 \%)$ & \\
\hline Sim & $32(57,1 \%)$ & $107(66,7 \%)$ & \\
\hline Posse de plano de saúde & & & 0,153 \\
\hline Não & $18(32,1 \%)$ & $68(43,0 \%)$ & \\
\hline Sim & $38(67,9 \%)$ & $90(57,0 \%)$ & \\
\hline
\end{tabular}

No modelo final ajustado, quando se controlou para os possíveis fatores de confusão, a maior prevalência de acesso ao fonoaudiólogo foi associada ao sexo feminino ( $\mathrm{RP}=1,09$; IC95\% 1,01$1,18)$ e a possuir alguma deficiência $(\mathrm{RP}=1,09$; IC95\% 1,03-1,17) (Tabela 2).

\section{Discussão}

Este estudo demonstrou que $26,2 \%$ da amostra pontuou a necessidade de consulta fonoaudiológica em algum momento, prioritariamente por queixas auditivas, seguida por queixas de fala. Destes indivíduos, 69,4\% conseguiram atendi- mento de forma privada e $19,6 \%$ a partir de convênio com plano de saúde.

O acesso ao serviço de saúde é complexo e relaciona-se, além da oferta, a capacidade de produzir serviços, de acordo com as necessidades de saúde percebidas pelo usuário, mediada por fatores individuais. Assim, fatores como queixas de saúde, originam demandas e, consequentemente, transformam-se no uso de serviços ${ }^{4,10}$.

As desigualdades socioeconômicas no acesso e na utilização do serviço de saúde estão relacionadas às características individuais, que afetam a necessidade e a busca por atendimento pelo indivíduo. Além disso, associam-se às variáveis contextuais, principalmente relacionadas às caracte- 
Tabela 2. Razões de prevalência (RP) bruta e ajustada em relação ao acesso dos cuidados em Fonoaudiologia. Porto Alegre, 2017.

\begin{tabular}{|c|c|c|c|c|}
\hline Variáveis & $\begin{array}{l}\text { RP bruta } \\
(\text { IC95\%) }\end{array}$ & p-valor & $\begin{array}{l}\text { RP ajustada } \\
(\text { IC } 95 \%)^{\star}\end{array}$ & p-valor \\
\hline \multicolumn{5}{|l|}{ Sexo } \\
\hline Masculino & 1 & - & 1 & - \\
\hline Feminino & $1,07(1,01-1,16)$ & 0,007 & $1,09(1,01-1,18)$ & 0,025 \\
\hline \multicolumn{5}{|l|}{ Idade } \\
\hline 18-39 anos & 1 & - & - & - \\
\hline $40-59$ anos & $0,95(0,88-1,03)$ & 0,243 & - & - \\
\hline 60 anos ou mais & $0,97(0,89-1,06)$ & 0,542 & - & - \\
\hline \multicolumn{5}{|l|}{ Cor/Raça } \\
\hline Branca & 1 & - & - & - \\
\hline Outra & $1,04(0,97-1,12)$ & 0,278 & $1,03(0,96-1,10)$ & 0,396 \\
\hline \multicolumn{5}{|l|}{ Situação conjugal } \\
\hline Em relacionamento & 1 & & - & - \\
\hline Solteiro & $0,99(0,93-1,06)$ & 0,787 & - & - \\
\hline \multicolumn{5}{|l|}{ Escolaridade } \\
\hline Até Ensino Fundamental & 1 & - & - & - \\
\hline Até Ensino Médio & $0,99(0,90-1,08)$ & 0,792 & - & - \\
\hline Ensino Superior ou mais & $0,99(0,91-1,09)$ & 0,916 & - & - \\
\hline \multicolumn{5}{|c|}{ Diagnóstico de doença crônica, mental ou física } \\
\hline Não & 1 & - & - & - \\
\hline Sim & $1,03(0,96-1,10)$ & 0,453 & - & - \\
\hline \multicolumn{5}{|l|}{ Possuir deficiência } \\
\hline Não & 1 & & 1 & \\
\hline Sim & $1,08(1,01-1,54)$ & 0,020 & $1,09(1,03-1,17)$ & 0,006 \\
\hline \multicolumn{5}{|c|}{ Utilizar a unidade de saúde do município } \\
\hline Não & 1 & - & 1 & - \\
\hline Sim & $0,95(0,88-1,02)$ & 0,169 & $0,96(0,89-1,04)$ & 0,324 \\
\hline \multicolumn{5}{|l|}{ Posse de plano de saúde } \\
\hline Não & 1 & - & 1 & - \\
\hline Sim & $1,05(0,98-1,12)$ & 0,141 & $1,04(0,97-1,12)$ & 0,284 \\
\hline
\end{tabular}

IC95\%: Intervalo de Confiança de 95\%. RP: Razão de Prevalência. *Ajuste para sexo, cor/raça, possuir deficiência, utilizar a unidade de saúde do município, posse de plano de saúde.

rísticas e a forma de organização do sistema de saúde, que, muitas vezes, acabam por reproduzir as desigualdades sociais no acesso aos serviços de saúde ${ }^{21}$. Uma melhor condição socioeconômica está associada a maior facilidade em obter cuidados em saúde do que uma pior condição. A desigualdade social no acesso à saúde tende a ser maior em países com sistemas de saúde privado do que em países com sistema universal ${ }^{22}$. O Brasil conta com um sistema de saúde organizado sob a forma de uma rede complexa de serviços complementares e competitivos, formando um sistema misto público-privado ${ }^{23}$ e este estudo demonstra que o acesso aos cuidados fonoaudiológicos acontece majoritariamente de forma privada ou conveniada a plano de saúde.
$\mathrm{O}$ acesso a uma consulta com um especialista específico, em diferentes países, tem ocorrido principalmente por indivíduos mais ricos ${ }^{11,12,24}$. Além disso, sabe-se que o acesso limitado ao fonoaudiólogo é um desafio a ser superado ${ }^{17,25,26}$, o que pode contribuir para a dificuldade no acesso deste profissional. No Brasil, faz apenas 36 anos que a Fonoaudiologia foi regulamentada como profissã $\mathrm{O}^{27}$, o que pode implicar em um número reduzido de profissionais, bem como ao pouco conhecimento da população sobre a sua atuação, não reconhecendo que alguns problemas de saúde possam ser reabilitados por tal profissional.

Dados da Organização Mundial da Saúde (OMS) indicam que 1,1 bilhão de pessoas no mundo podem desenvolver perda auditiva ${ }^{28}$, 
além disso, a OMS enfatiza que ações decisivas de prevenção e otimização dos serviços de reabilitação para deficientes auditivos devam ser priorizadas em todos os níveis de atenção ${ }^{29}$. Corrobora-se para o aumento de deficientes auditivos o envelhecimento populacional ${ }^{30}$, que está associado ao declínio da função auditiva ${ }^{31}$. Sendo assim, os achados deste estudo apontam que, atualmente, há maior procura pelos serviços fonoaudiológicos na área auditiva.

A deficiência é conceituada como perda ou anormalidade de estrutura anatômica, da função fisiológica ou psicológica, temporária ou permanente. As consequências que abrangem a comunicação, direta ou indiretamente, são a alteração da linguagem (da fala/escrita) e a da audição (ouvir), além das alterações músculo-esquelética e orgânicas (físicas), que, dependendo da área afetada, podem interferir na motricidade orofacial e/ou na deglutição, assim como a deficiência intelectual ${ }^{32}$. Destarte, os cuidados fonoaudiológicos são essenciais a essa população, como evidenciado neste estudo, com maior prevalência de acesso ao fonoaudiólogo por deficientes.

Pessoas com deficiência apresentam maior prevalência de doenças crônicas, maior risco de desenvolver doenças secundárias em função da deficiência e maior necessidade de tratamento não resolvida ${ }^{33-35}$. Relatam também dificuldade de utilização dos serviços de saúde em função das barreiras de acesso e de comunicação $0^{36,37}$. No presente estudo, entretanto, encontrou-se associação entre maior utilização dos serviços e deficiência. Uma possível explicação pode estar relacionada à maior carga de doença e gravidade dos casos aliada à necessidade de tratamento percebida pelas pessoas com deficiência, que influenciam a procura pelos serviços.

O uso regular de serviços de saúde é frequentemente maior entre mulheres do que homens em todas as faixas etárias ${ }^{38}$. De um modo geral, os problemas de saúde afetam tanto homens como mulheres semelhantemente; entretanto, há maior tendência de procura aos serviços pelas mulheres $^{39}$, o que pode justificar a maior prevalência de acesso ao fonoaudiólogo encontrado neste estudo.

Este estudo apresenta algumas limitações, entre elas ressalta-se o grande número de recusas, mesmo sendo realizadas entrevistas presenciais, amplamente treinadas, em horários diversos, com possibilidade de agendamentos sob preferência do morador, identificação clara do entrevistador, exposição e explanação do termo de consentimento, no interior dos domicílios, que se deram prioritariamente devido a insegurança e a repressão dos cidadãos diante a violência urbana. Entretanto, este é o único estudo de base populacional encontrado na literatura que evidencia a necessidade e acesso aos cuidados em Fonoaudiologia.

Conclui-se que o acesso ao fonoaudiólogo é mais frequente em consultórios particulares e em consultórios particulares conveniados ao plano de saúde, além disso, há acesso limitado ao fonoaudiólogo nos serviços públicos de saúde. A demanda fonoaudiológica é mais frequente para problemas de audição, seguida dos distúrbios da fala. Por fim, observa-se que as mulheres e os indivíduos com deficiência possuem maior prevalência de utilização do serviço fonoaudiológico. 


\section{Colaboradores}

RS Rech contribuiu para a concepção do projeto, coleta de dados, análise e interpretação dos dados, redação, revisão crítica do conteúdo intelectual e aprovação da versão final a ser publicada. PT Bulgarelli contribuiu para a concepção do projeto, coleta de dados, redação, revisão crítica do conteúdo intelectual e aprovação da versão final a ser publicada. AM Condessa contribuiu na redação, revisão crítica do conteúdo intelectual e aprovação da versão final a ser publicada. CM Santos contribuiu na redação, revisão crítica do conteúdo intelectual e aprovação da versão final a ser publicada. JB Hilgert contribuiu para a concepção do projeto, análise e interpretação dos dados, redação, revisão crítica do conteúdo intelectual e aprovação da versão final a ser publicada. BNG Goulart contribuiu para a concepção do projeto, análise e interpretação dos dados, redação, revisão crítica do conteúdo intelectual e aprovação da versão final a ser publicada.

\section{Agradecimentos}

Dra. Michelle Brown pela revisão final da versão em inglês. Ao Conselho Nacional de Desenvolvimento Científico e Tecnológico - CNPq pela bolsa de estudos de doutorado (RS Rech), assim como a bolsa produtividade CNPq de JB Hilgert e BNG Goulart.

\section{Referências}

1. Starfield B. Atenção Primária: equilíbrio entre necessidades de saúde, serviços e tecnologia. Brasília: Unesco, MS; 2002.

2. Andersen RM, Davidson PL. Improving access to care in america. Individual and Contextual Indicators. In Andersen RM, Davidson PL, Baumeister SE. Changing the US health care system: key issues in health services policy and management. San Francisco: Jossey-Bass; 2014. p. 33-65.

3. Souza ECFD, Rocha NDSPD, Uchoa ADC, Rocha PDM, Vilar RLA. Acesso e acolhimento na atenção básica: uma análise da percepção dos usuários e profissionais de saúde. Cad Saude Publica 2008; 24(1):s100-s110.

4. Travassos C, Martins M. A review of concepts in health services access and utilization. Cad Saude Publica 2004; 20(2):S190-S198.

5. Barata RB. Acesso e uso de serviços de saúde. São Paulo Perspect 2008; 22(2):19-29.

6. Almeida APSC, Nunes BP, Duro SMS, Facchini LA. Determinantes socioeconômicos do acesso a serviços de saúde em idosos: revisão sistemática. Rev Saude Publica 2017; 51:50.

7. Travassos C, Oliveira EXG, Viacava F. Desigualdades geográficas e sociais no acesso aos serviços de saúde no Brasil: 1998 e 2003. Cien Saude Colet 2006; 11(4):975-986.

8. Lima-Costa MF, Barreto S, Giatti L. A situação socioeconômica afeta igualmente a saúde de idosos e adultos mais jovens no Brasil? Um estudo utilizando dados da Pesquisa Nacional por Amostras de Domicílios PNAD/98. Cien Saude Colet 2002; 7(4):813-824.

9. Travassos C, Viacava F, Laguardia J. Health supplements in the Brazilian National Household Survey-PNAD. Rev Bras Epidemiol 2008; 11(1):98-112.

10. Stopa SR, Malta DC, Monteiro CN, Szwarcwald CL, Goldbaum M, Cesar CLG. Acesso e uso de serviços de saúde pela população brasileira, Pesquisa Nacional de Saúde 2013. Rev Saude Publica 2017; 51(Supl. 1):3s.

11. The Commonwealth Fund. 2010 International Health Policy Survey in Eleven Countries. New York: The Commonwealth Fund; 2010.

12. Centers for Medicare \& Medicaid Services. Physical therapy/occupational therapy/speech-language pathology services [página na Internet]. [acessado $2017 \mathrm{Mar}$ 10]. Disponível em: http://www.medicare.gov/coverage/pt-and-ot-and-speech-language-pathology

13. Viacava F, Bellido JG. Condições de saúde, acesso a serviços e fontes de pagamento, segundo inquéritos domiciliares. Cien Saude Colet 2016; 21(2):351-370.

14. Lima-Costa MF, Leite DM, Passos VC, Macinko J. Tendências em dez anos das condições de saúde de idosos brasileiros: evidências da Pesquisa Nacional por Amostra de Domicílios (1998, 2003, 2008). Cien Saude Colet 2011; 16(9):3689-3696.

15. Goulart BNG, Martins-Reis VO, Chiari BM. Household survey on self-declared communication disorders: study design and protocol. Audiol Commun Res 2015; 20(4):336-348. 
16. Schmidt JG, Goulart BNG, Martins-Reis VO. Prevalência de distúrbios fonoaudiológicos auto-relatados em uma população do sul do Brasil. Anais do XXIV Congresso Brasileiro de Fonoaudiologia. São Paulo, Brasil; 2016.

17. González-Fernández M, Huckabee ML, Doeltgen SH, Inamoto Y, Kagaya H, Saitoh E. Dysphagia Rehabilitation: Similarities and Differences in Three Areas of the World. Curr Phys Med Rehabil Reports 2014; 1(4):296-306.

18. Wall LR, Ward EC, Cartmill B, Hill AJ, Porceddu SV. Examining user perceptions of SwallowIT : A pilot study of a new telepractice application for delivering intensive swallowing therapy to head and neck cancer patients. J Telemed Telecare 2015; 23(1):53-59.

19. Bulgarelli PT. Acesso a atenção primária em Porto Alegre: perspectiva do usuário [dissertação]. Porto Alegre: Universidade Federal do Rio Grande do Sul; 2017.

20. Martins AB, D'Avila OP, Hilgert JB, Hugo FN. Atenção Primária a Saúde voltada as necessidades dos idosos: da teoria à prática. Cien Saude Colet 2014; 19(8):34033416.

21. Almeida APSC, Nunes BP, Duro SMS, Facchini LA. Determinantes socioeconômicos do acesso a serviços de saúde em idosos: revisão sistemática. Rev Saude Publica 2017; 51:50.

22. van Doorslaer E, Masseria C, Koolman X, OECD Health Equity Research Group. Inequalities in access to medical care by income in developed countries. CMAJ 2006; 174(2):177-183.

23. Paim J, Travassos C, Almeida C, Bahia L, Macinko J. The Brazilian health system: history, advances, and challenges. Lancet 2011;377(9779):1778-1797.

24. Suominen-Taipale AL, Koskinen S, Martelin T, Holmen J, Johnsen R. Differences in older adults' use of primary and specialist care services in two Nordic countries. Eur J Public Health 2004; 14(4):375-380.

25. Rech RS, Hugo FN, Schmidt JG, Goulart BNG, Hilgert JB. Presença fonoaudiológica no apoio matricial do Núcleo de Apoio à Saúde da Família no Brasil. Anais do XXIV Congresso Brasileiro de Fonoaudiologia. São Paulo, Brasil; 2016.

26. Bazzo LMF. Privação da oferta de serviços fonoaudiológicos no Sistema Único de Saúde (SUS) e a reforma do Estado: a mediação do debate. R Cimed Biol 2007; 6(2):190-196.

27. Brasil. Lei no 6.965, de 9 de dezembro de 1981. Dispõe sobre a regulamentação da profissão de Fonoaudiólogo, e determina outras providências. Diário Oficial da União 1981; $10 \mathrm{dez}$

28. World Health Organization (WHO). World Report on Disability [documento na Internet]. Genebra: WHO; 2011. [acessado 2017 Mar 10]. Disponível em: http:// www.who.int/disabilities/world_report/2011/report. pdf

29. Pienaar E, Stearn N. Self-reported outcomes of aural rehabilitation for adult hearing aid users in a South African context. S Afr J Commun Disord 2010; 57(1):4-13.
30. Banco Mundial. Envelhecendo em um Brasil mais vetho: implicações do envelhecimento populacional para o crescimento econômico, a redução da pobreza, as finanças públicas e a prestação de serviços. Washington: The World Bank; 2011.

31. Espmark AK, Rosenhall U, Erlandssom S, Steen B. The two faces of presbyacusis: hearing impairment and psychosocial consequences. Int J Audiol 2002; 41(2):125-135.

32. Chamie M. The status and use of the International Classification of Impairments, disabilities and Handicaps (ICIDH). World Health Stat Q 1990; 43:273-280.

33. Cavalheiro GN, Dornelles SS, Souza FRR, Padilha MI, Cavalcanti LFB, Fernandes AMBM. Perfil das pessoas com deficiência física e Políticas Públicas: a distância entre intenções e gestos. Cien Saude Colet 2016; 21(10):3131-3142.

34. Horner-Johnson W, Dobbertin K, Lee JC, Andresen EM. Disparities in chronic conditions and health status by type of disability. Disabil Health J 2013; 6(4):280-286.

35. Drum CE, Krahn G, Culley C, Hammond L. Recognizing and responding to the health disparities of people with disabilities. Calif J Health Promot 2005; 3(3):29-42.

36. Castro SS, Lefevvre F, Lefèvre AMC, Cesar CLG. Acessibilidade aos serviços de saúde por pessoas com deficiência. Rev Saude Publica 2011; 45(1):99-105.

37. Iezzoni LI, O’Day BL, Killeen M, Harker H. Communicating about health care: observations from persons who are deaf or hard of hearing. Annals Internal Medicine 2004; 140(50):356-362.

38. Green CA, Pope CR. Gender, psychosocial factors and the use of medical services: a longitudinal analysis. Soc Sci Med 1999; 48(10):1363-1372.

39. Pinheiro RS, Viacava F, Travassos C, Brito AS. Gênero, morbidade, acesso e utilização de serviços de saúde no Brasil. Cien Saude Colet 2002; 7(4):687-707.
Artigo apresentado em 17/10/2017

Aprovado em 02/07/2018

Versão final apresentada em 04/07/2018 
